\title{
Mitochondria, Amyloid $\beta$, and Alzheimer's Disease
}

\author{
Ryan D. Readnower, ${ }^{1,2}$ Andrew D. Sauerbeck, ${ }^{1,2}$ and Patrick G. Sullivan ${ }^{1,2}$ \\ ${ }^{1}$ Spinal Cord \& Brain Injury Research Center, University of Kentucky, Lexington, KY 40536, USA \\ ${ }^{2}$ Department of Anatomy and Neurobiology, University of Kentucky, Lexington, KY 40536, USA
}

Correspondence should be addressed to Patrick G. Sullivan, patsull@email.uky.edu

Received 20 December 2010; Accepted 25 January 2011

Academic Editor: Cesare Mancuso

Copyright ( 2011 Ryan D. Readnower et al. This is an open access article distributed under the Creative Commons Attribution License, which permits unrestricted use, distribution, and reproduction in any medium, provided the original work is properly cited.

\begin{abstract}
Hypometabolism is a hallmark of Alzheimer's disease (AD) and implicates a mitochondrial role in the neuropathology associated with $\mathrm{AD}$. Mitochondrial amyloid-beta $(\mathrm{A} \beta)$ accumulation precedes extracellular $\mathrm{A} \beta$ deposition. In addition to increasing oxidative stress, $A \beta$ has been shown to directly inhibit mitochondrial enzymes. Inhibition of mitochondrial enzymes as a result of oxidative damage or $\mathrm{A} \beta$ interaction perpetuates oxidative stress and leads to a hypometabolic state. Additionally, $\mathrm{A} \beta$ has also been shown to interact with cyclophilin $\mathrm{D}$, a component of the mitochondrial permeability transition pore, which may promote cell death. Therefore, ample evidence exists indicating that the mitochondrion plays a vital role in the pathophysiology observed in AD.
\end{abstract}

\section{Introduction}

The incidence of Alzheimer's disease (AD) in the US is expected to increase to as many as 13.2 million by 2050 [1]. $\mathrm{AD}$ pathology is characterized by the progressive accumulation of senile plaques (consisting of amyloid $\beta$-peptide, $A \beta$ ) and neurofibrillary tangles (consisting of aggregates of the microtubule-associated protein tau). Oxidative damage has been implicated to play an early role in the pathogenesis of $\mathrm{AD}$ [2]. In $\mathrm{AD}$ patients a significant decrease in energy metabolism is observed in the frontal and temporal lobes as indicated by in vivo positron emission tomography (PET) [3]. Correlated with this increase in oxidative damage and decrease in energy metabolism is a decrease in mitochondrial enzyme (cytochrome $c$ oxidase, pyruvate dehydrogenase, $\alpha$ ketoglutarate dehydrogenase) activity in AD patients [4-6].

Mitochondrial dysfunction has been shown to play a key role in age-related neurodegenerative diseases, such as Alzheimer's disease and Parkinson's disease [7]. Mitochondria produce the majority of ATP in cells and function to maintain $\mathrm{Ca}^{2+}$ homeostasis. Mitochondria produce ATP by coupling electron transfer to the pumping of protons across the inner mitochondrial membrane. However, electrons can escape the electron transport chain and reduce oxygen to form reactive oxygen species (ROS). Oxidative damage results from a disturbance in the ROS-antioxidant balance that favors oxidation. Oxidative damage to mitochondria may be especially relevant in neurodegenerative disease since mitochondria are regulators of both cellular metabolism and apoptosis [8].

\section{Mitochondrial Enzyme Oxidative Damage and ROS Production}

A hallmark of $\mathrm{AD}$ is hypometabolism which, importantly, precedes the clinical presentation of the disease $[9,10]$. Early studies utilizing PET indicated that brain metabolism throughout the cortex in $\mathrm{AD}$ patients is significantly lower than cortical metabolism in normal subjects [11,12]. Clinical data from PET studies have shown which areas of the brain are mostly affected by mild and moderate AD, such as the posterior cingulate cortex, parietotemporal cortex, and prefrontal association cortices [13]. Also decreased metabolism and synaptic loss have been shown to overlap in the frontal and middle temporal gyri [14-17].

Mitochondria sustain the activity of neurons by producing ATP via the electron transport system (ETC) (Figure 1). Complex I (NADH dehydrogenase) catalyzes the transfer of two electrons from NADH to coenzyme Q [18]. Complex III (ubiquinol-cytochrome $c$ oxidoreductase) catalyzes the transfer of electrons from coenzyme Q to cytochrome $c$. 
Complex IV (cytochrome $c$ oxidase) reduces oxygen to water. As electrons are transported through complexes I, III, and IV, protons are pumped into the inner membrane space, generating an electrochemical gradient. This store of energy is used to generate ATP via the ATP synthase. ROS production is linked to membrane potential $(\Delta \psi)$ such that a high $\Delta \psi$ promotes increased ROS production [9]. High $\Delta \psi$ results in altered redox potential of ETC carriers and an increase in the half-life time of ubisemiquinone leading to increased ROS production. Also, any damage to components of the ETC could lead to a stalling of reduced intermediates of the ETC which would increase the probability of an electron slipping and reducing $\mathrm{O}_{2}$ to form ROS.

Postmortem assessment of human $\mathrm{AD}$ brains has revealed increased levels of oxidative damage which coincides with impairments in metabolism and $\mathrm{A} \beta$ processing [19]. Since mitochondria are the primary source of cellular ROS production, it is at least conceivable that as an organism ages mitochondrial enzymes would be especially vulnerable to oxidative damage. Damage to mitochondrial enzymes would cause defects in electron transport and promote ROS production. Redox proteomic analysis has revealed that a number of mitochondrial proteins are oxidatively modified including VDAC, aconitase, GAPDH, and lactate dehydrogenase in $\mathrm{AD}$ patients [20].

The most documented reduction of mitochondrial enzyme activity in $\mathrm{AD}$ is the activity of complex IV [5, 21-23]. Since peroxidative damage of cardiolipin in mitochondrial membranes has been shown to affect the activity of complex IV; ROS-mediated damage of membranes is postulated to partly be responsible for $\mathrm{A} \beta$ inhibition of complex IV [24]. However, since antioxidants fail to fully protect mitochondria from amyloid beta toxicity, it has been hypothesized that $A \beta$ itself directly inhibits mitochondrial enzymes [24]. The $A \beta_{25-35}$ fragment has been shown to selectively inhibit complex IV [25]. The $A \beta_{25-35}$ fragment retains the residues required for aggregation and undergoes aggregation more rapidly than full-length $A \beta$. In another study, both the fulllength $A \beta_{1-42}$ and $A \beta_{25-35}$ fragment resulted in inhibition of complex IV, and $\mathrm{A} \beta_{25-35}$ raised the $K_{m}$ of complex IV for reduced cytochrome $c$ [26]. The authors concluded that $\mathrm{A} \beta$ may act as an inhibitor of one of the cytochrome $c$ binding sites of complex IV.

Additionally, $\alpha$-ketoglutarate dehydrogenase has been shown to have reduced activity in AD [27]. Recently we reported an age-dependent decrease in NADH-linked, complex I- driven respiration rate and an increase in mitochondrial ROS production in aged dogs [28]. Therefore, it is evident that cumulative oxidative damage over the lifespan of an organism can affect mitochondrial efficiency and leave neurons susceptible to cell death.

\section{Oxidation of Mitochondrial DNA}

It has been hypothesized that ongoing oxidative damage to mitochondrial DNA (mtDNA) may be the underlying mechanism for cellular senescence [29]. Since mtDNA repair mechanisms are limited and because mtDNA is situated in

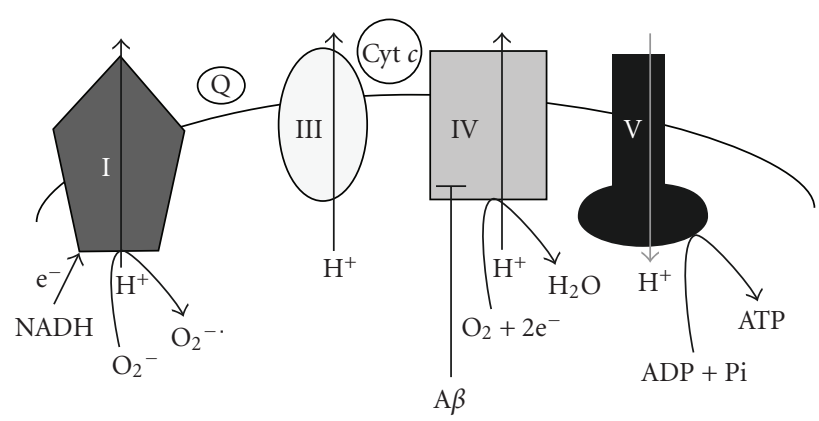

FIGURE 1: The relationship between amyloid- $\beta(\mathrm{A} \beta)$, mitochondrial electron transport chain (ETC), and superoxide $\left(\mathrm{O}_{2}^{-\bullet}\right)$ formation. As electrons are transferred through complexes I, III, and IV, protons are pumped into the inner membrane space, generating an electrochemical gradient. The energy stored is used to generate ATP via complex V (ATP synthase). Damage to components of the ETC can lead to a stalling of reduced intermediates which increases the probability of electrons slipping and reducing $\mathrm{O}_{2}$ to form superoxide. $\mathrm{A} \beta$ has been shown to directly inhibit complex IV which would lead to bioenergetic impairment and increased formation of reactive oxygen species.

close proximity to the site of ROS production, mtDNA is more vulnerable to oxidative damage than nuclear DNA [30]. With age, oxidation of mtDNA increases compared to nuclear DNA leading to an age-dependent accumulation of mtDNA mutations [31]. One recent study found that somatic mtDNA control region mutations are elevated in $\mathrm{AD}$ patients [32]. These mutations would lead to an overall reduction in mtDNA copy number which would result in a decrease in oxidative phosphorylation. In addition, a mutation that affects L-strand transcription was also discovered. This mutation inhibits complex I respiration which leads to increased ROS production, decreased membrane potential, and subsequent $\mathrm{Ca}^{2+}$ deregulation. The effects of these mutations may lead to opening of the mitochondrial permeability transition pore and subsequent neuronal death. Other evidence that oxidative damage to mtDNA could contribute to $\mathrm{AD}$ pathology is the observation that a risk factor for late-onset $\mathrm{AD}$ is a maternal history of $\mathrm{AD}$ [33]. This observation could be related to the fact that mitochondrial DNA is maternally inherited.

\section{Amyloid- $\beta$ : Cause or Effect of Mitochondrial Dysfunction}

$A \beta$ has been shown to accumulate in mitochondria from $\mathrm{AD}$ patients [34]. Altered processing of amyloid precursor protein (APP) which results in deposition of neurotoxic forms of $\mathrm{A} \beta$, known as the amyloid hypothesis of $\mathrm{AD}$, is believed to play a central role in the development of $\mathrm{AD}$ [35]. This hypothesis is supported by studies which show that immunization against $A \beta$ decreases amyloid levels and improves cognition in APP transgenic mice [36-38].

Intracellular and mitochondrial accumulation of $\mathrm{A} \beta$ likely precedes extracellular $A \beta$ deposition $[34,39,40]$. Recently $\mathrm{A} \beta$ has been shown to accumulate early (as young 
as $4 \mathrm{mo}$ ) and specifically in synaptic mitochondria [40]. We have shown that synaptic mitochondria have high levels of cyclophilin D (CypD) which makes them more susceptible to changes in synaptic $\mathrm{Ca}^{2+}$ [41]. Importantly, synaptic $\mathrm{Ca}^{2+}$ homeostasis is regulated by synaptic mitochondria, and susceptibility to $\mathrm{Ca}^{2+}$ would disrupt synaptic function [42]. CypD is a peptidyl-prolyl cis-trans isomerase located in the matrix and is a component of the mitochondrial permeability transition pore (mPTP). CypD translocates from the matrix to the MPTP and interacts with the adenine nucleotide translocase of the inner membrane to promote pore formation. Opening of the mPTP leads to a collapse in $\Delta \psi$ and release of proapoptotic molecules (i.e., cytochrome $\mathrm{C}$, Smac/Diablo, and apoptosis-inducing factor). A reduction in $\Delta \psi$ would lead to bioenergetic failure and subsequent synaptic failure ending in neuronal death. In fact, mitochondrial $\mathrm{A} \beta$ has been shown to interact with CypD, and CypD deficiency attenuates $A \beta$-induced mitochondrial stress [43]. This may indicate that because synaptic mitochondria have increased levels of, (i) cyclophilin D and (ii) $A \beta$, they are important in the pathogenesis of AD.

Mitochondrial dynamics are altered in $\mathrm{AD}$ patients, and mitochondrial fission has been shown to be more prevalent than fusion in $\mathrm{AD}$ [44]. This observation is supported by evidence indicating that the number of mitochondria is decreased in $\mathrm{AD}$ which corresponds with an increase in mitochondrial size [45]. APP overexpression, through $A \beta$ production, was shown to increase protein levels of proteins associated with fission (Fis1) and decreased protein levels of those involved in fusion (dynamin-like protein and OPA1) [46]. In addition $A \beta$ has been shown to cause oxidative damage to Drp1 which resulted in mitochondrial fission [47]. Altered mitochondrial dynamics may result in decreased mtDNA copy number which would result in defects in mitochondrial electron transport activity.

While the exact mechanism underlying APP mismetabolism is unclear, it appears that $A \beta$ production itself is least partially induced by oxidative stress [48]. $\mathrm{A} \beta$ can function both as an antioxidant and pro-oxidant [49]. At low concentrations (low-nanomolar) A $\beta$ remains monomeric and can function as an antioxidant. However, at higher concentrations, aggregation of $\mathrm{A} \beta$ produces $\mathrm{H}_{2} \mathrm{O}_{2}$. A $\beta$ induced oxidative stress and mitochondrial dysfunction has been demonstrated in AD models [50, 51]. In APP mutant mice that display increased levels of $\mathrm{A} \beta$, cognitionrelated brain regions have altered glucose metabolism [52]. Furthermore, mitochondria-targeted antioxidants have been shown to attenuate $\mathrm{A} \beta$-induced mitochondrial dysfunction [53]. Taken together, mitochondrial-induced oxidative stress may induce the production of $\mathrm{A} \beta$, which itself increases oxidative stress and impairs mitochondrial function and may provide a feed-forward loop that increases $A \beta$ levels.

\section{Conclusion}

In summary, since cerebral hypometabolism and inhibition of mitochondrial function have been demonstrated in $\mathrm{AD}$, mitochondria likely play a role in $\mathrm{AD}$ neuropathology. $\mathrm{A} \beta$ has been shown to both directly and indirectly impair mitochondrial function. Impairment of mitochondrial function increases ROS production which may further damage mitochondrial enzymes and mtDNA. Likely, mitochondrial dysfunction exacerbates the production of ROS and $A \beta$, which provides a feed-forward mechanism ultimately leading to $\mathrm{AD}$ pathology.

\section{References}

[1] L. E. Hebert, P. A. Scherr, J. L. Bienias, D. A. Bennett, and D. A. Evans, "Alzheimer disease in the US population: prevalence estimates using the 2000 census," Archives of Neurology, vol. 60, no. 8, pp. 1119-1122, 2003.

[2] M. A. Smith, C. A. Rottkamp, A. Nunomura, A. K. Raina, and G. Perry, "Oxidative stress in Alzheimer's disease," Biochimica et Biophysica Acta - Molecular Basis of Disease, vol. 1502, no. 1, pp. 139-144, 2000.

[3] M. P. Mattson, "Pathways towards and away from Alzheimer's disease," Nature, vol. 430, no. 7000, pp. 631-639, 2004.

[4] G. E. Gibson, H. Zhang, K. F. R. Sheu et al., " $\alpha$-ketoglutarate dehydrogenase in Alzheimer brains bearing the APP670/671 mutation," Annals of Neurology, vol. 44, no. 4, pp. 676-681, 1998.

[5] R. Castellani, K. Hirai, G. Aliev et al., "Role of mitochondrial dysfunction in Alzheimer's disease," Journal of Neuroscience Research, vol. 70, no. 3, pp. 357-360, 2002.

[6] J. P. Blass, "Brain metabolism and brain disease: is metabolic deficiency the proximate cause of Alzheimer dementia?" Journal of Neuroscience Research, vol. 66, no. 5, pp. 851-856, 2001.

[7] M. F. Beal, "Mitochondrial dysfunction in neurodegenerative diseases," Biochimica et Biophysica Acta, vol. 1366, no. 1-2, pp. 211-223, 1998.

[8] P. G. Sullivan, A. G. Rabchevsky, P. C. Waldmeier, and J. E. Springer, "Mitochondrial permeability transition in CNS trauma: cause or effect of neuronal cell death?" Journal of Neuroscience Research, vol. 79, no. 1-2, pp. 231-239, 2005.

[9] P. G. Sullivan and M. R. Brown, "Mitochondrial aging and dysfunction in Alzheimer's disease," Progress in NeuroPsychopharmacology and Biological Psychiatry, vol. 29, no. 3, pp. 407-410, 2005.

[10] J. P. Blass, R. K. F. Sheu, and G. E. Gibson, "Inherent abnormalities in energy metabolism in Alzheimer disease: interaction with cerebrovascular compromise," Annals of the New York Academy of Sciences, vol. 903, pp. 204-221, 2000.

[11] M. J. de Leon, S. H. Ferris, A. E. George et al., "Positron emission tomographic studies of aging and Alzheimer disease," American Journal of Neuroradiology, vol. 4, no. 3, pp. 568-571, 1983.

[12] E. Koss, R. P. Friedland, B. A. Ober, and W. J. Jagust, "Differences in lateral hemispheric asymmetries of glucose utilization between early- and late-onset Alzheimer-type dementia," American Journal of Psychiatry, vol. 142, no. 5, pp. 638-640, 1985.

[13] K. Herholz, E. Salmon, D. Perani et al., "Discrimination between Alzheimer dementia and controls by automated analysis of multicenter FDG PET," NeuroImage, vol. 17, no. 1, pp. 302316, 2002.

[14] S. W. Scheff and D. A. Price, "Alzheimer's disease-related synapse loss in the cingulate cortex," Journal of Alzheimer's Disease, vol. 3, no. 5, pp. 495-505, 2001. 
[15] S. W. Scheff and D. A. Price, "Synapse loss in the temporal lobe in Alzheimer's disease," Annals of Neurology, vol. 33, no. 2, pp. 190-199, 1993.

[16] G. S. Smith, M. J. De Leon, A. E. George et al., "Topography of cross-sectional and longitudinal glucose metabolic deficits in Alzheimer's disease: pathophysiologic implications," Archives of Neurology, vol. 49, no. 11, pp. 1142-1150, 1992.

[17] S. Minoshima, B. Giordani, S. Berent, K. A. Frey, N. L. Foster, and D. E. Kuhl, "Metabolic reduction in the posterior cingulate cortex in very early Alzheimer's disease," Annals of Neurology, vol. 42, no. 1, pp. 85-94, 1997.

[18] D. G. Nicholls, "Mitochondrial function and dysfunction in the cell: its relevance to aging and aging-related disease," International Journal of Biochemistry and Cell Biology, vol. 34, no. 11, pp. 1372-1381, 2002.

[19] F. Mangialasche, M. C. Polidori, R. Monastero et al., "Biomarkers of oxidative and nitrosative damage in Alzheimer's disease and mild cognitive impairment," Ageing Research Reviews, vol. 8, no. 4, pp. 285-305, 2009.

[20] R. Sultana and D. A. Butterfield, "Oxidatively modified, mitochondria-relevant brain proteins in subjects with Alzheimer disease and mild cognitive impairment," Journal of Bioenergetics and Biomembranes, vol. 41, no. 5, pp. 441-446, 2009.

[21] S. J. Kish, C. Bergeron, A. Rajput et al., "Brain cytochrome oxidase in Alzheimer's disease," Journal of Neurochemistry, vol. 59, no. 2, pp. 776-779, 1992.

[22] W. D. Parker, C. M. Filley, and J. K. Parks, "Cytochrome oxidase deficiency in Alzheimer's disease," Neurology, vol. 40, no. 8, pp. 1302-1303, 1990.

[23] I. Maurer, S. Zierz, and H. J. Möller, "A selective defect of cytochrome c oxidase is present in brain of Alzheimer disease patients," Neurobiology of Aging, vol. 21, no. 3, pp. 455-462, 2000.

[24] X. Wang, B. Su, G. Perry, M. A. Smith, and X. Zhu, "Insights into amyloid- $\beta$-induced mitochondrial dysfunction in Alzheimer disease," Free Radical Biology and Medicine, vol. 43, no. 12, pp. 1569-1573, 2007.

[25] L. Canevari, J. B. Clark, and T. E. Bates, “ $\beta$-Amyloid fragment 25-35 selectively decreases complex IV activity in isolated mitochondria," FEBS Letters, vol. 457, no. 1, pp. 131-134, 1999.

[26] C. S. Casley, L. Canevari, J. M. Land, J. B. Clark, and M. A. Sharpe, " $\beta$-Amyloid inhibits integrated mitochondrial respiration and key enzyme activities," Journal of Neurochemistry, vol. 80, no. 1, pp. 91-100, 2002.

[27] S. Sorbi, E. D. Bird, and J. P. Blass, "Decreased pyruvate dehydrogenase complex activity in Huntington and Alzheimer brain," Annals of Neurology, vol. 13, no. 1, pp. 72-78, 1983.

[28] E. Head, V. N. Nukala, K. A. Fenoglio, B. A. Muggenburg, C. W. Cotman, and P. G. Sullivan, "Effects of age, dietary, and behavioral enrichment on brain mitochondria in a canine model of human aging," Experimental Neurology, vol. 220, no. 1, pp. 171-176, 2009.

[29] J. Miquel, "An update on the mitochondrial-DNA mutation hypothesis of cell aging," Mutation Research, vol. 275, no. 3-6, pp. 209-216, 1992.

[30] S. Z. Imam, B. Karahalil, B. A. Hogue, N. C. Souza-Pinto, and V. A. Bohr, "Mitochondrial and nuclear DNA-repair capacity of various brain regions in mouse is altered in an agedependent manner," Neurobiology of Aging, vol. 27, no. 8, pp. 1129-1136, 2006.

[31] J. Sastre, F. V. Pallardó, J. García De La Asunción, and J. Viña, "Mitochondria, oxidative stress and aging," Free Radical Research, vol. 32, no. 3, pp. 189-198, 2000.
[32] P. E. Coskun, J. Wyrembak, O. Derbereva et al., "Systemic mitochondrial dysfunction and the etiology of Alzheimer's disease and down syndrome dementia," Journal of Alzheimer's Disease, vol. 20, supplement 2, pp. S293-S310, 2010.

[33] L. Mosconi, V. Berti, R. H. Swerdlow, A. Pupi, R. Duara, and M. de Leon, "Maternal transmission of Alzheimer's disease: prodromal metabolic phenotype and the search for genes," Human Genomics, vol. 4, no. 3, pp. 170-193, 2010.

[34] M. Manczak, T. S. Anekonda, E. Henson, B. S. Park, J. Quinn, and P. H. Reddy, "Mitochondria are a direct site of $\mathrm{A} \beta$ accumulation in Alzheimer's disease neurons: implications for free radical generation and oxidative damage in disease progression," Human Molecular Genetics, vol. 15, no. 9, pp. 1437-1449, 2006.

[35] J. Hardy and D. J. Selkoe, "The amyloid hypothesis of Alzheimer's disease: progress and problems on the road to therapeutics," Science, vol. 297, no. 5580, pp. 353-356, 2002.

[36] C. Janus, J. Pearson, J. McLaurin et al., "A $\beta$ peptide immunization reduces behavioural impairment and plaques in a model of Alzheimer's disease," Nature, vol. 408, no. 6815, pp. 979982, 2000.

[37] D. Morgan, D. M. Diamond, P. E. Gottschall et al., “A $\beta$ peptide vaccination prevents memory loss in an animal model of Alzheimer's disease," Nature, vol. 408, no. 6815, pp. 982985, 2000.

[38] D. M. Wilcock, A. Rojiani, A. Rosenthal et al., "Passive immunotherapy against $\mathrm{A} \beta$ in aged APP-transgenic mice reverses cognitive deficits and depletes parenchymal amyloid deposits in spite of increased vascular amyloid and microhemorrhage," Journal of Neuroinflammation, vol. 1, no. 1, p. 24, 2004.

[39] O. Wirths, G. Multhaup, C. Czech et al., "Intraneuronal A $\beta$ accumulation precedes plaque formation in $\beta$-amyloid precursor protein and presenilin-1 double-transgenic mice," Neuroscience Letters, vol. 306, no. 1-2, pp. 116-120, 2001.

[40] H. Du, L. Guo, S. Yan, A. A. Sosunov, G. M. McKhann, and S. S. Yan, "Early deficits in synaptic mitochondria in an Alzheimer's disease mouse model," Proceedings of the National Academy of Sciences of the United States of America, vol. 107, no. 43, pp. 18670-18675, 2010.

[41] K. K. Naga, P. G. Sullivan, and J. W. Geddes, "High cyclophilin D content of synaptic mitochondria results in increased vulnerability to permeability transition," Journal of Neuroscience, vol. 27, no. 28, pp. 7469-7475, 2007.

[42] M. F. Galindo, I. Ikuta, X. Zhu, G. Casadesus, and J. Jordán, "Mitochondrial biology in Alzheimer's disease pathogenesis," Journal of Neurochemistry, vol. 114, no. 4, pp. 933-945, 2010.

[43] H. Du, L. Guo, F. Fang et al., "Cyclophilin D deficiency attenuates mitochondrial and neuronal perturbation and ameliorates learning and memory in Alzheimer's disease," Nature Medicine, vol. 14, no. 10, pp. 1097-1105, 2008.

[44] D. J. Bonda, X. Wang, G. Perry, M. A. Smith, and X. Zhu, "Mitochondrial dynamics in alzheimers disease: opportunities for future treatment strategies," Drugs and Aging, vol. 27, no. 3, pp. 181-192, 2010.

[45] K. Hirai, G. Aliev, A. Nunomura et al., "Mitochondrial abnormalities in Alzheimer's disease," Journal of Neuroscience, vol. 21, no. 9, pp. 3017-3023, 2001.

[46] X. Wang, B. Su, S. L. Siedlak et al., "Amyloid- $\beta$ overproduction causes abnormal mitochondrial dynamics via differential modulation of mitochondrial fission/fusion proteins," Proceedings of the National Academy of Sciences of the United States of America, vol. 105, no. 49, pp. 19318-19323, 2008. 
[47] D. H. Cho, T. Nakamura, J. Fang et al., “ $\beta$-Amyloid-related mitochondrial fission and neuronal injury," Science, vol. 324, no. 5923, pp. 102-105, 2009.

[48] M. Recuero, T. Muñoz, J. Aldudo, M. Subías, M. J. Bullido, and F. Valdivieso, "A free radical-generating system regulates APP metabolism/processing," FEBS Letters, vol. 584, no. 22, pp. 4611-4618, 2010.

[49] A. Kontush, "Amyloid- $\beta$ : an antioxidant that becomes a prooxidant and critically contributes to Alzheimer's disease," Free Radical Biology and Medicine, vol. 31, no. 9, pp. 1120-1131, 2001.

[50] V. Rhein, G. Baysang, S. Rao et al., "Amyloid-beta leads to impaired cellular respiration, energy production and mitochondrial electron chain complex activities in human neuroblastoma cells," Cellular and Molecular Neurobiology, vol. 29, no. 6-7, pp. 1063-1071, 2009.

[51] J. Yao, R. W. Irwin, L. Zhao, J. Nilsen, R. T. Hamilton, and R. D. Brinton, "Mitochondrial bioenergetic deficit precedes Alzheimer's pathology in female mouse model of Alzheimer's disease," Proceedings of the National Academy of Sciences of the United States of America, vol. 106, no. 34, pp. 14670-14675, 2009.

[52] J. C. Dodart, C. Mathis, K. R. Bales, S. M. Paul, and A. Ungerer, "Early regional cerebral glucose hypometabolism in transgenic mice overexpressing the V717F $\beta$-amyloid precursor protein," Neuroscience Letters, vol. 277, no. 1, pp. 49-52, 1999.

[53] M. Manczak, P. Mao, M. J. Calkins et al., "Mitochondriatargeted antioxidants protect against amyloid- $\beta$ toxicity in Alzheimer's disease neurons," Journal of Alzheimer's Disease, vol. 20, supplement 2, pp. S609-S631, 2010. 


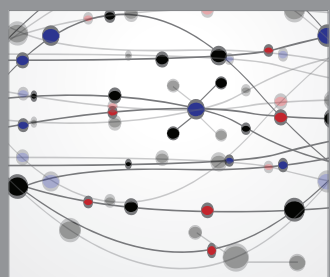

The Scientific World Journal
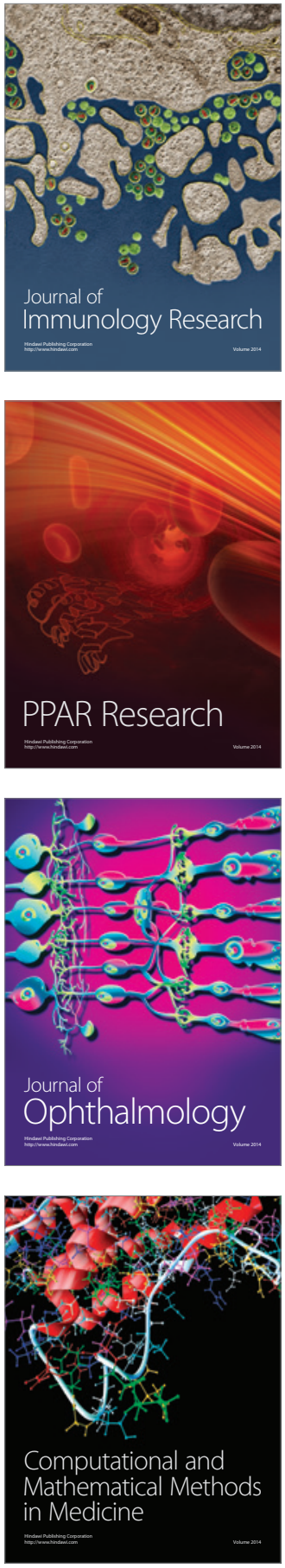

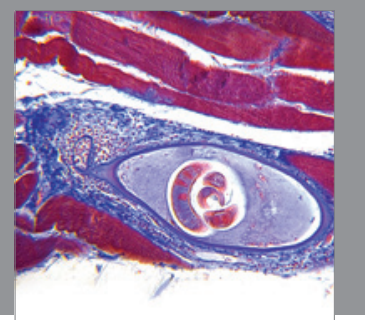

Gastroenterology

Research and Practice
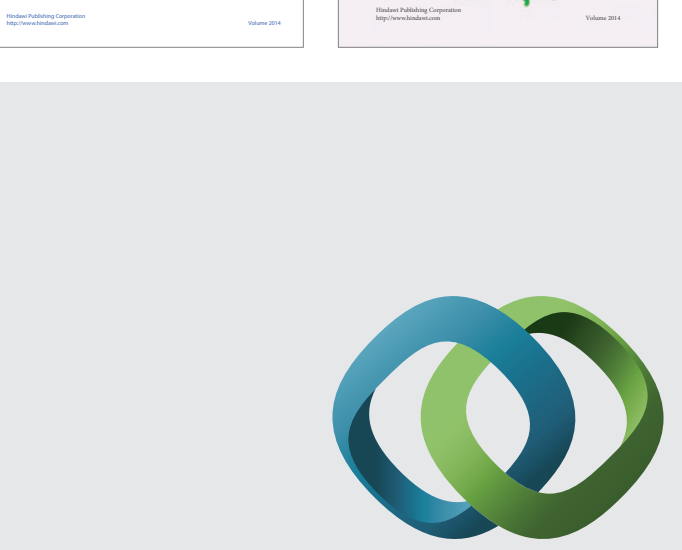

\section{Hindawi}

Submit your manuscripts at

http://www.hindawi.com
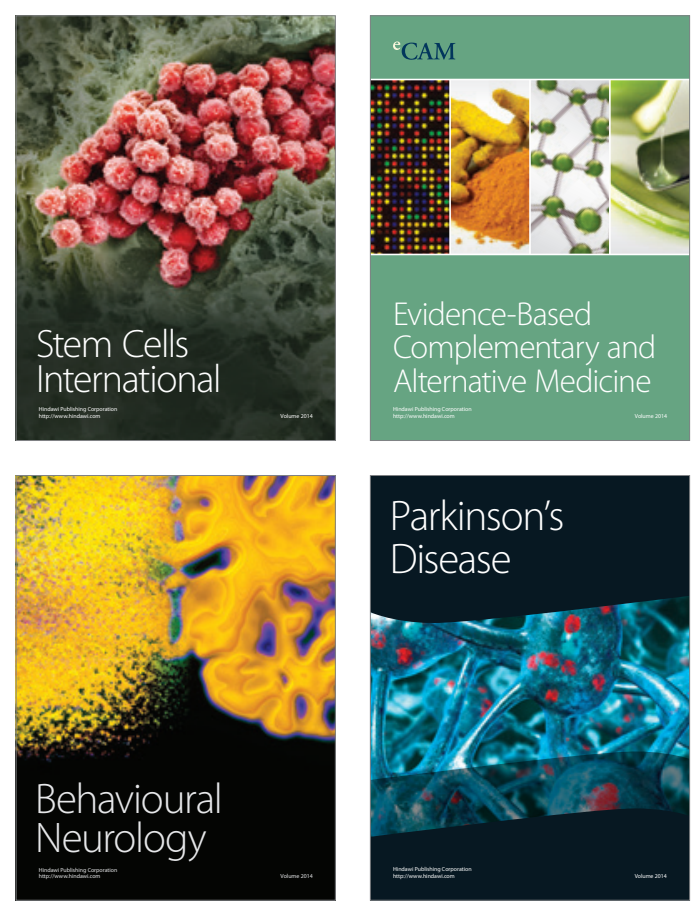

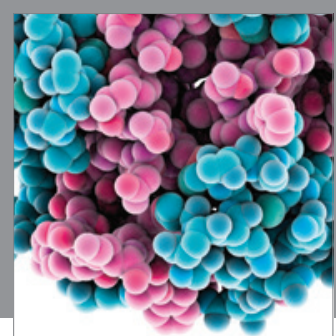

Journal of
Diabetes Research

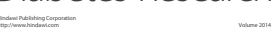

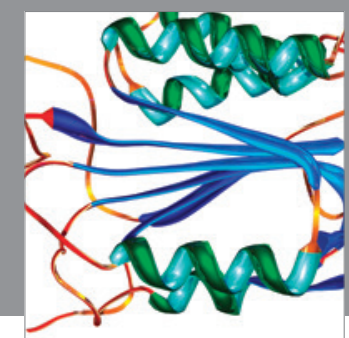

Disease Markers
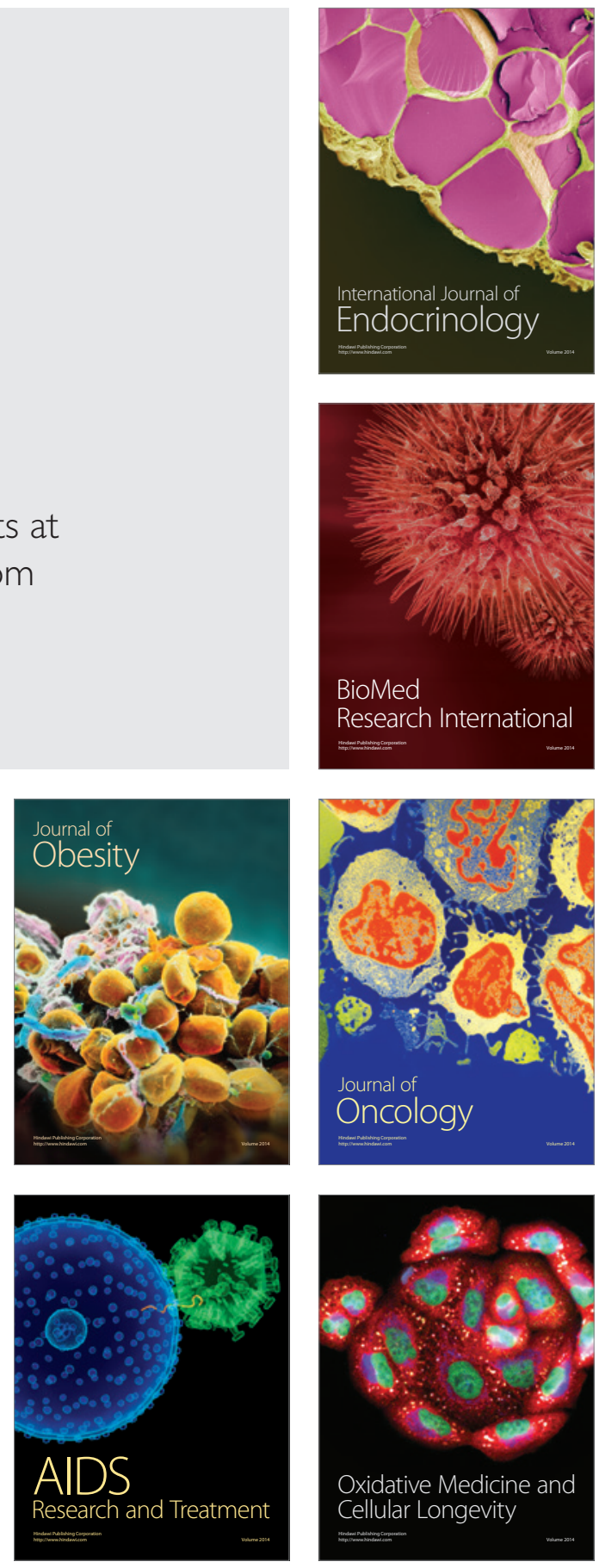